\title{
Psychometric properties and normative data of the Patient Health Questionnaire (PHQ-9) in the general population of South Korea
}

\section{Cheolmin Shin, Changsu Han Korea University College of Medicine, Seoul, Republic of Korea}

\section{Background}

Background: $\mathrm{PHQ}-9$ has been standardized in several populations and is widely used in clinical practice and health care. However, it has not been appropriately standardized in the Korean general population and no normative data has been presented.

Objectives: The aims of the study were to present normative data of the PHQ-9 and to standardize the PHQ-9 in the general population of South Korea.

\section{Methods}

Methods: We used nationwide cross-sectional survey data in South Korea from 2014 to 2016 and total 10756 individuals aged over 19 years were enrolled in the study. The survey questionnaires included the PHQ-9, EuroQol-5 dimension (EQ-5D) and demographic characteristics. The construct validity and internal consistency of the $\mathrm{PHQ}-9$ in general population were analyzed. To explore factor structure of PHQ-9, the exploratory and confirmatory factor analysis (CFA) was performed. The normative data of the PHQ-9 were generated according to gender and different age categories.

\section{Results}

The mean score of $P H Q-9$ was higher for women than for men [3.35 (SD, 3.36) vs. 2.19 (SD, 4.31)]. In both gender, the PHQ-9 mean score showed a U-shape relationship with the age group. The correlation coefficient between the sum of PHQ-9 scores and the EQ-5D index was -0.44 , which was moderate. The best fitting model was the two-factor model in our sample. $T$
The best fitting model was the two-factor model in our sample. This result was conformed by CFA (Fit Indices : $\mathrm{Cmin} / \mathrm{df}=52.89$, $\mathrm{TLI}=0.922, \mathrm{CFI}=0.944, \mathrm{RMSEA}=0.070)$. In the PHQ-9 questionnaires, item 4 which ask fatigue was most frequently scored more than 1 point. For most items except item 4 and item 7 , the response rate was significantly different by age group. Item 1 through item 7 tended to be more responsive in younger ages, and item 8 through item 9 tended to be more responsive in older ages.

\section{Discussion}

In Korean general population, PHQ-9 was a reliable and valid tool for assessing depression. PHQ-9 has a two-factor structure and In the general population of Korea, PHQ-9 was a two-factor structure, which is comparable to that of one factor structure in representative population of Germany.

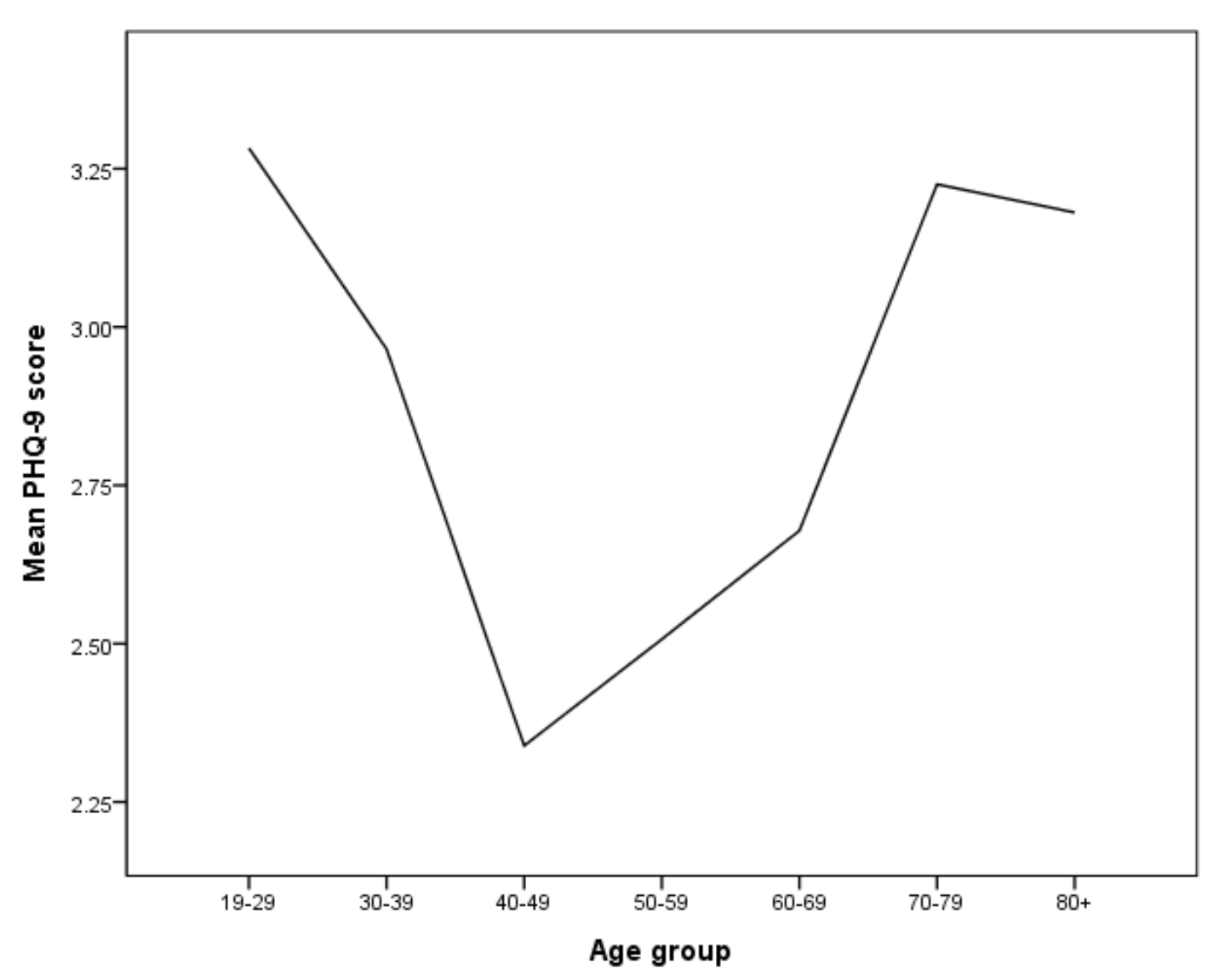

Figure 1. Mean score of PHQ-9 according to age group

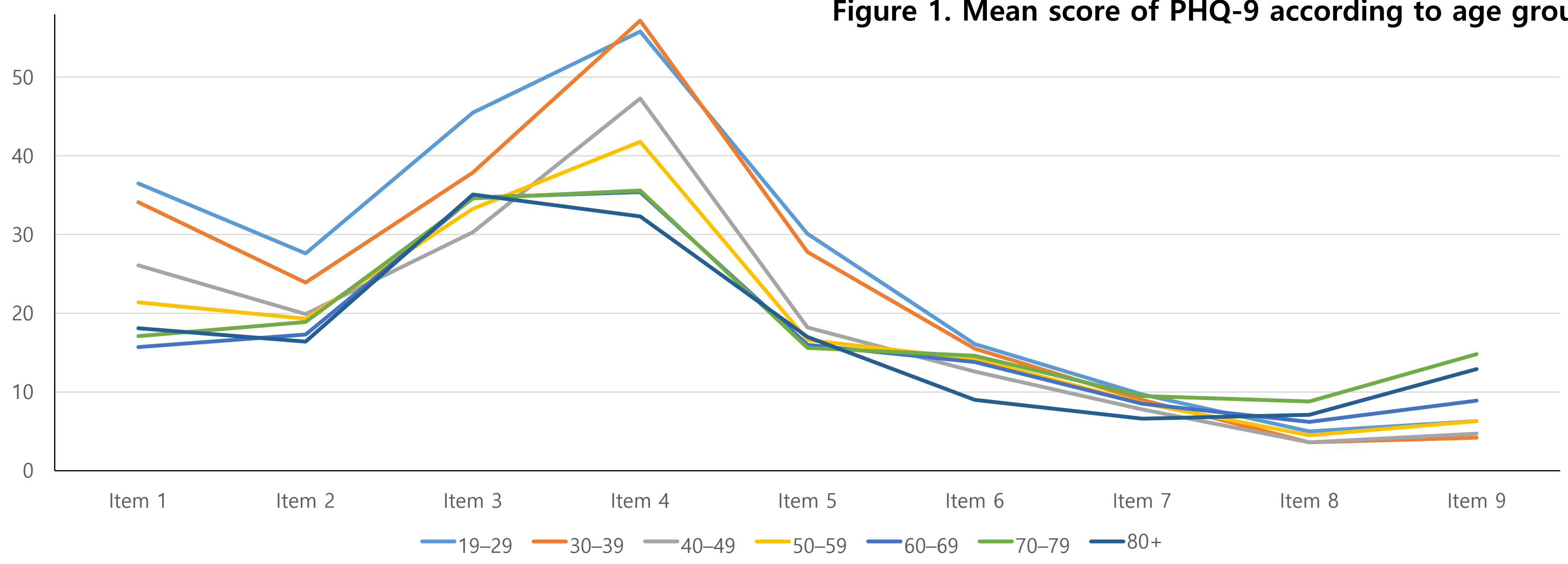

Figure 2. Response rate by PHQ-9 item by age group 